\title{
Perceptions of Pap Screening in a Context of HPV Vaccination
}

\author{
Spring Cooper ${ }^{*}$, Louise Bezzina ${ }^{2}$, Hayden Fletcher ${ }^{3}$
}

\begin{abstract}
Objectives: A development in the prevention of cervical cancer in women in Australia was the introduction of the human papillomavirus (HPV) vaccine, which was made available to young women as part of a national school-based vaccination program in 2007. Despite this advancement, Pap screening is still required to provide optimal protection from cervical cancer. The current study aimed to explore what young women know about HPV, HPV vaccination, and Pap screening guidelines. It further aimed to identify barriers and facilitators young women perceive to participating in screening, as well as factors influencing these perceptions. Materials and Methods: Semi-structured interviews were conducted with 12 women who had been in school during the Australian HPV vaccination program and offered HPV vaccine, aged 18-25, until saturation around key themes was reached. Transcripts were analysed, letting themes emerge from the data.

Results: Key barriers and facilitators to pap screening were identified, as well as factors affecting the salience of these barriers and facilitators.

Conclusions: Findings have implications for the development of interventions to increase screening adherence and reduce the incidence of cervical cancer in this population.

Keywords: Papillomavirus vaccines, Primary prevention, Papanicolaou test, Health education, Reproductive health
\end{abstract}

\section{Introduction}

Cervical cancer is second most common cancer affecting women and the third most common cause of cancer mortality in women worldwide (1). Cervical cancer is caused by infection with the sexually transmitted human papillomavirus (HPV), of which there are over 120 types (2). It has been estimated that over $80 \%$ of sexually active females will be infected with HPV in their lifetimes, most in their mid to late teens, 20s, and early 30s (3). While infection is often harmless and clears spontaneously, persistent infection with high-risk types can lead to the development of cervical cancer (4).

\section{Human Papillomavirus Vaccine}

A recent advancement in the prevention of cervical cancer was the introduction of the quadrivalent prophylactic HPV vaccine. When administered prior to exposure, vaccination prevents infection with HPV 16 and 18, the HPV types responsible for 70\%-80\% of cervical cancer globally (5). It also protects against HPV 6 and 11, the HPV types responsible for the development of the majority of genital warts cases (6). Between 2007-2009, Australia implemented a National HPV Vaccination catch-up program in which the 3 -dose vaccination was administered free to all women aged between 12-26 years of age. Estimates of vaccination coverage for young women in the catch-up program are 64\%, 59\%, and 53\% for doses 1, 2, and 3 respectively (7). The HPV vaccine is currently free in Australia as part of an ongoing schoolbased vaccination program for adolescents aged 12-13 years.

\section{Cervical Cancer Screening}

While the HPV vaccine is effective in protecting against 2 high-risk types of HPV, up to $30 \%$ of cervical cancer cases worldwide are caused by oncogenic types of HPV not included in the vaccine (8). These forms of HPV can only be detected, and any consequences subsequently treated, through cervical cancer screening. The combination of vaccination and regular screening provides optimal protection from cervical cancer, reducing the lifetime risk by $94 \%(9)$.

\section{Reduction in Adherence to Screening Guidelines}

Since 1996, participation in 2-year screening has declined in Australia, with the largest decrease observed for women aged 20-24 (10). In 1996, 62.4\% of the target age group of 20-69 years old were screened; in the period from 20112012 , it was $58 \%$ (10). However, at $42.8 \%$, participation was lowest for women aged 20-24 (down from a baseline of $50 \%$ in 1996) (10). The lower and consistently declining rates of screening in young women are particularly 
important as this age group having the highest detection rate of cervical abnormalities (10).

The introduction of the National HPV Vaccination Program in Australia raised concerns that the declining rates of screening participation in young women would be exacerbated: of 298 general practitioners surveyed during the Program, 25\% anticipated that the HPV vaccine would reduce participation in cervical screening (10). However, a survey conducted in 2009 showed that $96 \%$ of vaccinated women in Victoria aged 18-28 years knew that screening was still suggested after vaccination (11-13).

Recent Australian data suggests that knowledge of the need for screening despite vaccination did not translate to behaviour. A cross-sectional analysis of data from the Victorian Cervical Cytology Registry and the National HPV Vaccination Program Register for women in Victoria found that women vaccinated against HPV as part of the catch up program participated in significantly less screening compared to their unvaccinated counterparts (11). It was found that $47.7 \%$ of unvaccinated women aged $20-24$, compared to $37.6 \%$ of vaccinated women, participated in 2-year screening (14). A similar difference was observed for women aged 25-29, with $58.7 \%$ of unvaccinated women attending the 2-year screening compared to $45.2 \%$ of vaccinated women (14). There is therefore a compelling need to investigate reasons for the disparity in screening between vaccinated and unvaccinated Australian women.

The Current Study: Rationale and Aims

Although levels of knowledge and attitudinal and practical barriers to cervical screening are well documented (12), it is not clear if or how these differ for vaccinated and unvaccinated young Australian women in influencing screening participation; specifically, women who are in this cohort of women offered HPV vaccination at school, regardless of whether they accepted the vaccine. An increased understanding of these factors will assist with the provision of adequate information and service delivery needs. This provision is expected to increase adherence to cervical screening guidelines, which is critical for the reduction in the incidence of cervical cancer.

The current study is therefore aimed to explore what young women know about HPV, HPV vaccination, and Pap screening guidelines. It further aimed to identify barriers and facilitators young women perceive to participating in screening, as well as factors influencing these perceptions.

\section{Materials and Methods}

\section{Participants}

Theoretical sampling was employed so that we could recruit a total of 12 young women at university aged 1825 (average age of 21) that represented a diverse range of socioeconomic and ethnic backgrounds as well as women that had and had not ever had Pap screening and had and had not received HPV vaccination (all women were part of the cohort offered vaccination, but we recruited women that had and had not elected to accept vaccination). These sources included advertisements placed in university common areas, announcements in summer classes, and on social media platforms. Sampling continued until conceptual saturation was achieved (13).

\section{Data Collection}

Qualitative semi-structured interviews were based on the Health Belief Model and Theory of Planned Behaviour: for example, we asked about benefits and barriers to Pap screening; intentions to get a Pap screen; and ideas about outcome expectations (14). Interviews were conducted at The University of Sydney or via Skype video calling. The following topics were explored in relation to HPV vaccination and screening: screening uptake, knowledge of screening recommendations; attitudes to screening; screening history and intentions; sources of information about screening and vaccination; knowledge deficits about screening and vaccination; relation of vaccination to screening; desired content of information; and recommendations to increase screening uptake.

\section{Data Analysis}

Interview data were digitally recorded, transcribed and recurring themes and patterns were identified. We used an iterative process to modify themes as we collected data; first analysis began after the first four interviews were conducted. One researcher (LB) began analysis and came up with the first set of themes. The next set of themes was established as the other researchers (HF and SC) reviewed new data. As new data was gathered, analyses continued to test the earlier/previous set of concepts, patterns and relationships. This process was continued until a coherent and robust framework emerged. The final results were agreed upon by all researchers.

\section{Results}

The core themes presented in this paper are the young women's identified barriers and facilitators to participating in regular pap screening. Supporting themes were self-concept and aspects of understanding and knowledge that influence the salience of these barriers and facilitators. Based on these themes, participants provided recommendations. See Figure 1 for a pictorial representation of these themes and responses.

\section{Participant Characteristics}

The 12 women recruited represented a range of screening and vaccination experiences and attitudes/intentions as shown in Table 1.

\section{Barriers}

While there were several barriers identified to Pap screening, the most frequently cited barriers fell into five categories: psychological, physical, time, location, and economic barriers. Characteristic quotes for each of these 


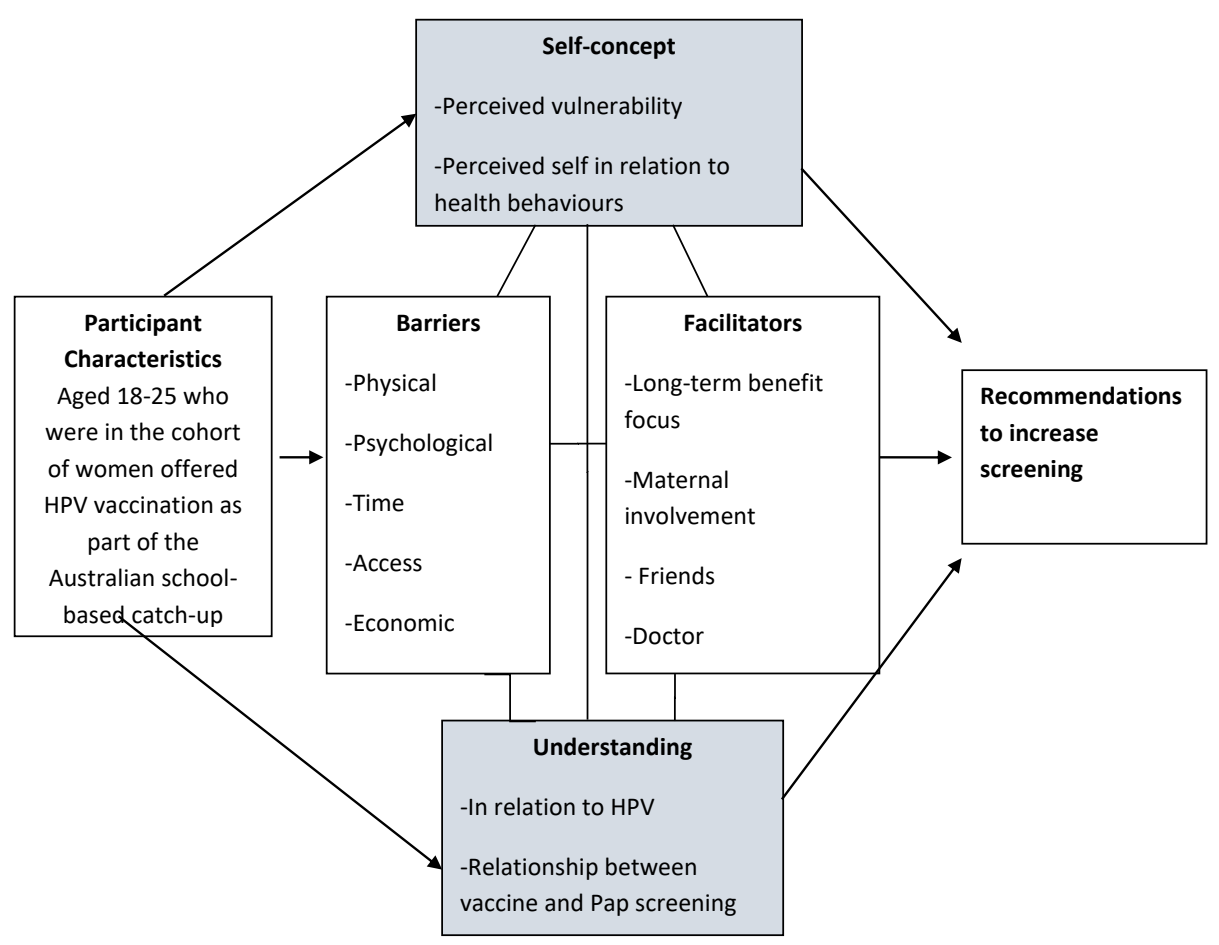

Figure 1. Pictorial Representation of Themes.

Boxes in the centre represent the key barriers and facilitators of Pap screening. Coloured boxes represent self-concept and knowledge/ understanding which were key factors affecting the salience of the barriers and facilitators. These are not meant to be causal arrows. Rather the blue boxes are merely factors that seemed to characterize the barriers and facilitators, but that were influenced by who this specific population was (part of the school-based program) and that coloured their following recommendations.

barriers are shown in Table 2.

Psychological barriers refer to self-limiting beliefs a person may have that affects their behaviour. This was the most frequently cited barrier amongst participants, and they described it as being a strong barrier. The vulnerability that participants felt was evident in their responses, and this was present in women who had and had not been screened previously.

Physical barriers were less emphasised, but were important to some women. The majority of participants were not concerned with the physical pain with most participants describing only mild concern about discomfort. The main difference in perceptions of physical barriers was between women who had and had not been screened before. Participants who had not had Pap screening expressed concern that the procedure would be very painful. In contrast, participants who had participated in screening responded that they had been surprised at experiencing less discomfort than they had anticipated.

Table 1. Participant Characteristics

\begin{tabular}{llll}
\hline & $\begin{array}{l}\text { Had a Pap } \\
\text { Screen }\end{array}$ & $\begin{array}{l}\text { Never Had a } \\
\text { Pap Screen }\end{array}$ & Total \\
\hline $\begin{array}{l}\text { Vaccinated (at least one } \\
\text { dose) }\end{array}$ & $\mathrm{n}=8$ & $\mathrm{n}=3$ & $\mathrm{n}=11$ \\
$\begin{array}{l}\text { Non-vaccinated } \\
\text { Total }\end{array}$ & $\mathrm{n}=0$ & $\mathrm{n}=1$ & $\mathrm{n}=1$ \\
\hline
\end{tabular}

The majority of participants cited lack of time as a barrier to Pap screening. They talked about their studies at University, their family commitments, and wanting to spend time with friends as things that limited them from making appointments for screening.

Several participants who had not had a Pap screen expressed confusion about where to get a Pap screen. Participants who had previously had a Pap screen reported that their friends were unaware of where to get Pap screening and that they had helped friends in the past to find a suitable location

Another frequently cited barrier was concern about the potential cost involved. This was a hypothetical, as the young women did not know if there would be any cost involved, or how much it might be. But the potential that there may be a cost was a barrier for making an appointment.

\section{Facilitators}

The most common facilitators for regular Pap screening included focus on the long-term benefit, maternal support and discussion, discussion with friends, relationship with doctor and previous experience. Characteristic quotes for each of these barriers are shown in Table 3.

Many of the participants who engaged in regular Pap screening tended to focus conversation on the long-term benefit of Pap screening, particularly prevention of cervical cancer. These participants expressed that although the 
Table 2. Five Categories of Cited Barriers

\begin{tabular}{|c|c|}
\hline Perceived Barrier & Characteristic quotes \\
\hline Psychological & $\begin{array}{l}\text { "I think it's more the psychological part than the physical part because you build it up in your head" (P5). } \\
\text { "I guess it's more the psychological...being very vulnerable" (P10). } \\
\text { "You've gotta be naked, and someone's like looking at that, and that's mentally uncomfortable" (P1). }\end{array}$ \\
\hline Physical & $\begin{array}{l}\text { "I'm afraid it will hurt really badly... just the way my body is I think it would be very difficult and painful... I've convinced myself } \\
\text { that it would be so awful and cold and spiky... I think it's gonna hurt" (P7). } \\
\text { "I kind of walked out of there surprised, like is that it? I just thought it would be painful..." (P11). }\end{array}$ \\
\hline Time & $\begin{array}{l}\text { "Time is probably would have been the biggest thing... it's always difficult to take time out and go and do these things" (P10). } \\
\text { "I'm sure there are a lot of people out there that are the same as me who have schedules too full" (P9). } \\
\text { "I'm not uncomfortable in getting it I just haven't gotten around to doing it yet" (P8). }\end{array}$ \\
\hline Access & $\begin{array}{l}\text { "I don't know whether you'd have to go to a specialist... I don't really know who's qualified to do that test" (P7). } \\
\text { "I think just knowing where to get one... I think being aware of where to get one would probably help" (P8). } \\
\text { "I think a lot of people don't really know where to get things done... I think I told about } 6 \text { people that there was a family } \\
\text { planning clinic in [town name removed] that they didn't know about" (P6). }\end{array}$ \\
\hline Economic & $\begin{array}{l}\text { "A lot of it for me and my friends... it's also cost. You've got to pay a consultation fee for the doctor and a lot of them are } \\
\text { studying and don't have that sort of money... I think cost is a big barrier" (P12). } \\
\text { "It's more an accessibility thing or a worry about cost, like having to pay a gap" (P4). }\end{array}$ \\
\hline
\end{tabular}

Table 3. Common Facilitators for Regular Pap Screening

\begin{tabular}{|c|c|}
\hline Facilitator & Characteristic quotes \\
\hline $\begin{array}{l}\text { Focus on long-term } \\
\text { benefit }\end{array}$ & $\begin{array}{l}\text { "Yes it's uncomfortable but cancer's a hell of a lot more uncomfortable" (P2). } \\
\text { "It's not the most comfortable thing but if you prevent yourself from getting cancer why wouldn't you do anything you } \\
\text { could do" (P11). } \\
\text { "It's for my benefit and that's what I've gotta keep reminding myself despite that I am so awkward about that kinda stuff" } \\
\text { (P5). } \\
\text { 'It's certainly awkward for } 20 \text { minutes or so but what's that in a lifetime basically?" (P11). }\end{array}$ \\
\hline Maternal support & $\begin{array}{l}\text { "If I didn't have mum telling me you should go and do these things... I wouldn't have made that choice" (P10). } \\
\text { "We have a pretty open and honest relationship...she gave me a run down on what the procedure would entail" (P10). } \\
\text { "She gave me options of where to go" (P4) } \\
\text { "Probably I would just ask my mum and see who she goes to" (P9). }\end{array}$ \\
\hline $\begin{array}{l}\text { Discussion with } \\
\text { friends }\end{array}$ & “It sorta took a friend to say that 'you should really just go'” (P4). \\
\hline Doctor & "I saw a doctor regularly and built a relationship with and felt comfortable enough to feel able to ask for it" (P11). \\
\hline $\begin{array}{l}\text { Previous Pap screen } \\
\text { experience }\end{array}$ & $\begin{array}{l}\text { "I think after having one I wouldn't hesitate to have my next one...you realise it's not that bad at all" (P4). } \\
\text { "Next time I would be a lot more comfortable because l'd know what to expect" (P6). }\end{array}$ \\
\hline
\end{tabular}

screening may be uncomfortable they were able to put the experience in perspective when they compared it to longterm negative outcomes of neglecting screening, including cervical cancer.

Another key facilitator appeared to be maternal involvement and support. Many participants reported that their mother had been a key factor in promoting them to get a Pap smear. Maternal involvement was also influential in gaining information about the process of Pap screening such as what would be involved and locations in which screening could be performed. Lack of maternal involvement, or restricted discussion on the subject, appeared to be of detriment to Pap screening behaviour.

Discussion with friends also appeared to be a key facilitator to screening in many participants. Participants discussed how they had been influenced by their friends urging them to get a Pap smear. One participant recounted her friend's experience of cervical cancer, and that this experience was an impetus for her to partake in regular screening. Further, she said that her friend actively urged others to undertake in screening. Conversation around Pap screening appeared to be less common in participants who had not had a Pap screen and were overdue.

Doctor-patient relationship was another key facilitator for attending Pap screenings, with participants stating that having a regular doctor made them more likely to feel comfortable asking for the screening.

Having had a Pap screen previously was a facilitator for engaging in regular Pap screening. Participants reported that after their first Pap smear, they felt more confident about undertaking another.

Self-concept

A key factor affecting the salience of the barriers and facilitators discussed is self-concept. Two aspects of 
participant's self concept appeared particularly important: Perceived vulnerability and their perception of self in relation to health behaviours. Quotes demonstrating these factors are shown in Table 4.

Perceived vulnerability describes participants' beliefs regarding how likely they were to be affected by HPV. Some participants responded that HPV was not something they perceived to affect people in their age range whereas other participants were highly concerned about developing cervical cancer, usually due to personal or family history of cancer.

Perception of self in relation to health behaviours describes how participants viewed their ability to protect their health. Several participants viewed themselves as proactive in maintaining their health and took pride in this behaviour. In contrast, participants who had not engaged in Pap screening described themselves as lazy and berated themselves for this.

Understanding of General and In-depth HPV Knowledge Knowledge and understanding surrounding HPV was another factor affecting the salience of the barriers and facilitators. Two aspects were of importance: knowledge about what HPV is and understanding the relationship between vaccination and screening. Quotes demonstrating these factors are shown in Table 5.

The majority of participants were able to identify that HPV was a virus that could lead to genital warts and cervical cancer. They were also able to explain that it was transmitted sexually and that there were different types. However, some participants were neither aware of what
HPV is nor of the potential consequences of having HPV. All participants, when asked, stated that both regular Pap screening and the HPV vaccination were necessary for optimal protection from HPV. However, while one could explain, with a fairly high accuracy, why both Pap screening and vaccination were necessary, others were unsure of the reason for having both.

\section{Recommendations}

A number of recommendations for encouraging young women to adhere to the Pap screening guidelines emerged from discussion with participants. Participants' recommendations included increasing knowledge and improving education, reducing stigma, and improving accessibility. Participants also made recommendations in relation to advertising approaches. A sample of these recommendations is shown in Table 6 .

\section{Discussion}

The current study is, to our knowledge, the first to explore and compare the knowledge, attitudes, and behaviours related to screening of young Australian women were involved in the National HPV Vaccination catch up program (regardless of vaccination status). All young women possessed knowledge that Pap screening was necessary regardless of vaccination status. Despite this knowledge, the young women varied in their adherence to screening guidelines. Further exploration revealed that the intention-behaviour gap between intending to partake in screening and actually adhering to the specified guidelines was influenced by a range of barriers and facilitators. The

Table 4. Self-concept

\begin{tabular}{ll}
\hline Self-concept & Characteristic quotes \\
\hline & "No one thinks it's important when they're young" (P1). \\
"There are ads... often older people...that's not me I must not be in an at-risk population... must not be a worry" (P4). & "I have a friend who had never had a pap screen before and her doctor made her get one at 22 and she had cancer. \\
& She was quite shocked...she didn't think that a 22 year old could get it" (P2). \\
"I know that given my history having had cancer it's something I should be doing" (P5) & "I try and keep on top of them. There's been a history of cancer in my family" (P10) \\
"I always thought I was quite on top of keeping my sexual health" (P5). & "I've always been one to try to look after myself" (P2). \\
"I was quite impressed with myself... I probably felt more in control of my health and more aware... I felt like I'd done & something positive for my health" (P4). \\
"“I'm just lazy. It's not at the forefront of my mind" (P1) \\
in relation to health \\
behaviours
\end{tabular}

Table 5. Knowledge and Understanding of HPV

\begin{tabular}{|c|c|}
\hline Knowledge & Characteristic quotes \\
\hline Knowledge about HPV & $\begin{array}{l}\text { “...because I don't really know what the disease is... what it affects...I don't really know" (P8) } \\
\text { "Not a whole lot I feel like I’m very uneducated on the subject...I genuinely know like literally nothing” (P6). }\end{array}$ \\
\hline $\begin{array}{l}\text { Knowledge of relationship } \\
\text { between vaccination and } \\
\text { Pap screening }\end{array}$ & $\begin{array}{l}\text { "I think you would but I don't know why. It just feels like you probably should do it anyway" (P8). } \\
\text { "The vaccination isn't 100\%. It doesn't give you an 'oh I've done that' tick'... there's always a chance that things could } \\
\text { be picked up in your Pap smear that the vaccination doesn't prevent" (P10). } \\
\text { "She [sister] was wondering why it was still necessary considering she's already had the vaccines. I told her a doctor } \\
\text { told me it was only preventing infection from } 3 \text { of the HPV so you could still get it in other forms" (P3). }\end{array}$ \\
\hline
\end{tabular}


Table 6. Recommendations

\begin{tabular}{|c|c|}
\hline Recommendation & Characteristic quotes \\
\hline $\begin{array}{l}\text { Increase knowledge and } \\
\text { improve education }\end{array}$ & $\begin{array}{l}\text { "Maybe more education in schools...I didn't learn about it in school" (P1). } \\
\text { "I definitely think there's not a lot of education in general... My [health class] information was very much lacking in } \\
\text { this area. I feel like maybe Universities or TAFE (Technical And Further Education) should have some sort of easier } \\
\text { accessible forms of information" (P6). } \\
\text { "An information booklet... just more information...I think when you have something invasive you want to have } \\
\text { more information than for something non invasive" (P4). } \\
\text { "A friendly pamphlet just explaining what would happen and what the experience would be like. I also didn't even } \\
\text { really know what the process would be or what she was checking or the way they check for things. I would have } \\
\text { appreciated knowing that" (P6). } \\
\text { "I'd like to know that it's not one size fits all...more acknowledgement that it is an unfamiliar and scary } \\
\text { process"(P7). }\end{array}$ \\
\hline Reduce stigma & $\begin{array}{l}\text { "I think it's definitely still stigmatised as being a weird or negative thing which I think a lot of aspects of women's } \\
\text { bodies are" (P6). } \\
\text { "It needs to be a bit more open in discussion... It does still have a bit of a stigma around it" (P5). }\end{array}$ \\
\hline Accessibility & $\begin{array}{l}\text { "I remember they had those big trailers for breast screening women... if there were more mobile facilities like that } \\
\text { people might be more obliged to take some time out of their day to do it" (P9). }\end{array}$ \\
\hline Advertising approaches & $\begin{array}{l}\text { "YouTube seems to be a good platform these days" (P9). } \\
\text { "University bus stops...that would be quite effective. Even if they don't want to do it it's kinda in their } \\
\text { consciousness" (P8). } \\
\text { "Maybe in a magazine...put it in Dolly magazine" (P1). } \\
\text { "I'm very uncomfortable with it being on TV...my father's like 'urgh do we have to watch this'... I know I shouldn't } \\
\text { say it's gross but it's gross" (P1). }\end{array}$ \\
\hline
\end{tabular}

salience of these barriers and facilitators was affected by self-concept and level of knowledge surrounding HPV and why screening remained necessary. These findings have important implications for future cervical cancer prevention interventions among both vaccinated and unvaccinated young women.

Participants identified several barriers to adherence to Pap screening guidelines. These included psychological, physical, time, location, and economic barriers. These barriers to cervical screening in young women are consistent with those found in the literature (15). The most prominent barrier to screening in the current sample was the anticipation, or previous experience, of psychological discomfort arising from both discussing screening with a health professional and undertaking screening. Interestingly, this finding conflicts with previous evidence which suggests that younger women are more likely to report practical barriers to screening, such as time restrictions, relative to attitudinal barriers (16). While practical barriers such as time and location were common in the current sample, they were not as prominent. However, an interesting point to note is that many participants who were overdue for Pap screening and cited time as a major barrier to screening were willing to meet to partake in the interview for the current study. The time allocated to this interview was approximately the amount of time a Pap screening appointment would take. This presents a contradiction between the participant's quoted barrier of time and their actual behaviour, suggesting the time barrier may not be as central a barrier as they self reported.

Facilitators to adherence to Pap screening guidelines included focusing on the long-term benefit of screening; namely, prevention of cervical cancer, maternal support and discussion, discussion with friends, relationship with their doctor, and whether or not they had undertaken a Pap screen in the past. These findings are in line with the broader literature that has documented the importance of social norms and open discussion surrounding screening behaviour in adherence to screening guidelines (17).

The salience of these barriers and facilitators varied between participants and was found to subsequently influence screening behaviour. One factor affecting the salience was participant's self-concept in relation to perceived vulnerability and perception to self in relation to health behaviours. Participant who expressed a perceived vulnerability to cervical cancer, particularly due to personal or family history, were more likely to give barriers to screening a low importance. Further, participants who perceived themselves as proactive in maintaining their health generally were more likely to take pride in their screening behaviour and similarly placed a lower emphasis on barriers to screening throughout discussion.

A second factor affecting the salience of barriers and facilitators was participant's knowledge and understanding of HPV and the relationship between the vaccination and Pap screening. This is in line with previous research that has documented limited knowledge surrounding HPV and the HPV vaccination in young Australian women. A study involving 130 adolescent girls demonstrated that there was a limited understanding of HPV, how it was transmitted, and the relationship between HPV and cervical cancer (18). The study also found a lack of knowledge surrounding 
what the HPV vaccine protects against, how it works, and the connection between the vaccine and screening (18). Participants in the current sample who had a low level of understanding surrounding HPV did not know that it could lead to cervical cancer and/or genital warts. Thus, the perceived barriers seemed to gain greater salience for less knowledgeable participants, as they were less able to weigh up the costs and benefits of screening in the same way as more knowledgeable participants. Further, while all participants were aware that screening was necessary despite vaccination status, only a portion of participants could articulate that this was that the vaccination protects against only a subset of the HPV types and that screening was necessary to detect infection with other types. This in turn affected the salience of perceived barriers and facilitators to screening.

Findings from the current study can be used to inform the development of interventions to increase screening and vaccination adherence. Successful interventions should aim to increase the salience of facilitators and reduce the salience of barriers to health protective behaviours. Findings suggest that this salience will be affected by targeting young women's self concept and increasing the knowledge of HPV and the role of vaccination and screening.

There are some limitations to consider in generalising the current study. The self-selection for participation in the study meant that the sample was not representative. Those who volunteered to participate may have had a greater interest in HPV, health and vaccination and consequently the current study results may reflect the views of more informed health consumers. The knowledge and understanding related to HPV and Pap screening may therefore be even lower in the overall population of young women than in the current sample.

Cervical cancer is one of the most common forms of cancer affecting young women and an increased understanding of how to prevent and manage incidence in the population is critical. The current study identified that young Australian women who had been vaccinated against HPV, and had not been vaccinated against HPV, have a good understanding of Pap screening guidelines and the requirement for regular screening. Importantly, it identified several barriers and facilitators to screening which affected the behaviour that arose from the intention to screen based on guideline knowledge. Factors affecting the salience of these perceived barriers and facilitators were also identified. The current findings have implications for the development of interventions to ensure that adherence to Pap screening guidelines is maintained in both vaccinated and unvaccinated Australian women.

\section{Conflict of Interests}

Authors declare that they have no conflict of interests.

\section{Ethical Issues}

This research was conducted at the University of Sydney and was approved by the University of Sydney Human Research Ethics Committee, approval number 2014/922.

\section{Financial Support}

Sydney Medical School's Summer Research Scholarship program supported Louise Bezzina in her work on this project.

\section{References}

1. Parkin DM, Bray F, Ferlay J, Pisani P. Global cancer statistics, 2002. CA Cancer J Clin. 2005;55(2):74-108.

2. de Villiers EM, Gunst K, Stein H, Scherubl H. Esophageal squamous cell cancer in patients with head and neck cancer: Prevalence of human papillomavirus DNA sequences. Int J Cancer. 2004;109(2):253-258. doi:10.1002/ijc.11685

3. Russell M, Raheja V, Jaiyesimi R. Human papillomavirus vaccination in adolescence. Perspect Public Health. 2013;133(6):320-324. doi:10.1177/1757913913499091

4. Saslow D, Solomon D, Lawson HW, et al. American Cancer Society, American Society for Colposcopy and Cervical Pathology, and American Society for Clinical Pathology screening guidelines for the prevention and early detection of cervical cancer. CA Cancer J Clin. 2012;62(3):147-172. doi:10.3322/ caac. 21139

5. Stanley MA. Human papillomavirus (HPV) vaccines: prospects for eradicating cervical cancer. J Fam Plann Reprod Health Care. 2004;30(4):213-215. doi:10.1783/0000000042177162

6. Ball SL, Winder DM, Vaughan K, et al. Analyses of human papillomavirus genotypes and viral loads in anogenital warts. J Med Virol. 2011;83(8):1345-1350. doi:10.1002/jmv.22111

7. Brotherton JM, Liu B, Donovan B, Kaldor JM, Saville M. Human papillomavirus (HPV) vaccination coverage in young Australian women is higher than previously estimated: independent estimates from a nationally representative mobile phone survey. Vaccine. 2014;32(5):592-597. doi:10.1016/j. vaccine.2013.11.075

8. Karanam B, Jagu S, Huh WK, Roden RB. Developing vaccines against minor capsid antigen L2 to prevent papillomavirus infection. Immunol Cell Biol. 2009;87(4):287-299. doi:10.1038/icb.2009.13

9. Goldie SJ, Grima D, Kohli M, Wright TC, Weinstein M, Franco E. A comprehensive natural history model of HPV infection and cervical cancer to estimate the clinical impact of a prophylactic HPV-16/18 vaccine. Int J Cancer. 2003;106(6):896-904. doi:10.1002/ ijc. 11334

10. Brotherton JM, Leask J, Jackson C, McCaffery K, 
Trevena LJ. National survey of general practitioners' experience of delivering the National Human Papillomavirus Vaccination Program. Sex Health. 2010;7(3):291-298. doi:10.1071/sh09135

11. Budd AC, Brotherton JM, Gertig DM, Chau T, Drennan KT, Saville M. Cervical screening rates for women vaccinated against human papillomavirus. Med J Aust. 2014;201(5):279-282.

12. Glasgow RE, Whitlock EP, Valanis BG, Vogt TM. Barriers to mammography and Pap smear screening among women who recently had neither, one or both types of screening. Ann Behav Med. 2000;22(3):223228.

13. Patton MQ. Qualitative research and evaluation methods. Thousand Oaks, CA: Sage; 2002.

14. Ajzen I. The theory of planned behavior. Organ Behav Hum Decis Process. 1991;50(2):179-211. doi:10.1016/0749-5978(91)90020-T

15. Siahpush M, Singh GK. Sociodemographic predictors of pap test receipt, currency and knowledge among Australian women. Prev Med. 2002;35(4):362-368.

16. Waller J, Jackowska M, Marlow L, Wardle J. Exploring age differences in reasons for nonattendance for cervical screening: a qualitative study. Bjog. 2012;119(1):26-32. doi:10.1111/j.14710528.2011.03030.x

17. Duffett-Leger LA, Letourneau NL, Croll JC. Cervical cancer screening practices among university women. J Obstet Gynecol Neonatal Nurs. 2008;37(5):572-581. doi:10.1111/j.1552-6909.2008.00276.x

18. Cooper Robbins SC, Bernard D, McCaffery K, Brotherton J, Garland S, Skinner SR. "Is cancer contagious?": Australian adolescent girls and their parents: making the most of limited information about HPV and HPV vaccination. Vaccine. 2010;28(19):3398-3408. doi:10.1016/j. vaccine. 2010.02 .078

(c) 2018 The Author (s); This is an open-access article distributed under the terms of the Creative Commons Attribution License (http://creativecommons.org/licenses/by/4.0), which permits unrestricted use, distribution, and reproduction in any medium, provided the original work is properly cited. 